\title{
Agentes Comunitários de Saúde: perspectivas, atividades preventivas e vigilância em saúde bucal da criança
}

\author{
Community Healthcare Agents: perspective, preventive \\ and surveillance activities in oral care of children
}

\author{
Agentes Comunitarios de Salud: perspectivas, actividades preventivas y de \\ vigilancia de la salud oral de los niños \\ André Luiz Marçal TERRERI \\ Daniela Pereira LIMA \\ Danielle PORTINHO \\ Mariângela Monteiro de Melo BALTAZAR \\ Marina BERTI \\ Curso de Odontologia - Universidade Estadual do Oeste do Paraná (UNIOESTE), 85819-110 - Cascavel (PR), Brasil
}

\begin{abstract}
Resumo
Introdução: O profissional Agente Comunitário de Saúde (ACS) surgiu na década de 90 com o objetivo de desenvolver atividade de prevenção de doenças e promoção de saúde junto aos domicílios da comunidade na qual atua. Dentre as suas ações, os ACS têm um papel importante na divulgação de informações sobre a saúde bucal. Objetivo: Descrever a percepção e atuação dos ACS, em relação à saúde bucal nas Unidades de Saúde da Família do município de Francisco Beltrão-PR. Método: A pesquisa foi realizada na forma de investigação de campo por abordagem quantitativa. Um questionário estruturado foi aplicado à 56 ACS pertencentes a 09 unidades de saúde com Equipes de Saúde Bucal (ESBs). Resultados: Raramente os ACS realizam atividades preventivas e de vigilância à saúde bucal durante suas visitas domiciliares. Destes, 71,4\% nunca receberam capacitação e demonstraram baixo conhecimento sobre cárie, gengivite e fluorose dentária. Outros 58,9\% nunca receberam acompanhamento da ESB em visitas domiciliares. Conclusão: Os resultados demonstram que a ESB pouco se articula com o trabalho dos agentes comunitários, dificultando mudanças no modelo de atenção à saúde bucal ofertado na estratégia de saúde da família do município.
\end{abstract}

Descritores: Promoção da Saúde; Saúde Bucal; Agentes Comunitários de Saúde; Educação em Saúde; Saúde da Família.

\begin{abstract}
Introduction: Community Health Agents (CHA) emerged in the 1990s with the goal of developing disease prevention activities and promotion of health with the community in which it operates. Among its action, ACS play an important role in the dissemination of information about oral health. Objective: Describe the perception and performance of the CHA in relation to oral health in the Family Health Units of the city of Francisco Beltrão - PR. Method: The research was a field investigation, using a quantitative approach. A structured questionnaire was applied to $56 \mathrm{CHA}$ belonging to 09 health units with Oral Health Teams (OHT). Results: The CHA practically do not carry out preventive and oral health surveillance activities during their home visits. $71.4 \%$ of CHAs never received training and showed low knowledge about caries, gingivitis and dental fluorosis. Other 58.9\% of CHAs never received follow-up from the oral health team during the home visits. Conclusion: The results show that the oral health team does not articulate with the work of community agents, making it difficult to change the model of oral health care offered in the family health strategy of the municipality. Descriptors: Health Promotion; Oral Health; Community Health Workers; Health Education; Family Health.
\end{abstract}

\begin{abstract}
Resumen
Introducción: Profesionales Agentes Comunitarios de Salud (ACS) surgió en los años 90 con el objetivo de desarrollar la actividad de prevención de enfermedades y promoción de la salud, junto con la comunidad de cuidado en el hogar en el que opera. Entre sus acciones, la ACS tiene un papel importante en la difusión de información sobre la salud oral. Objetivo: Describir la percepción y el rendimiento de la AEC, en relación con la salud bucal en las unidades de salud de la municipalidad de la familia Francisco Beltrao-PR. Método: La investigación se realizó en forma de investigación de campo por el enfoque cuantitativo. Un cuestionario estructurado se aplicó a 56 ACS pertenecientes a 09 unidades de salud con equipos de salud bucal (ESB). Resultados: En raras ocasiones ACS realizan actividades de prevención y vigilancia de la salud bucal durante sus visitas a los hogares. De estos, el 71,4\% nunca ha recibido capacitación y demostrado escaso conocimiento sobre la caries, la gingivitis y la fluorosis dental. Otro 58,9\% nunca recibió el control de la ESB en las visitas a domicilio. Conclusión: Los resultados muestran que el bit ESB está vinculada con el trabajo de los trabajadores de la comunidad, lo que dificulta los cambios en el modelo de atención a la salud bucal que se ofrece en la estrategia de salud de la familia municipal.

Descriptores: Promoción de la Salud; Salud Bucal; Agentes Comunitarios de Salud; Educación en Salud; Salud de la Familia.
\end{abstract}

\section{INTRODUÇÃO}

Fazer um histórico do trabalho no núcleo familiar resgata os anos 1987, quando se iniciou a implantação do Programa de Agentes de Saúde, no Ceará ${ }^{1}$, uma experiência bem-sucedida que estimulou o Ministério da Saúde (MS) a propor, em âmbito nacional o Programa de Agentes Comunitários de Saúde, no ano de 1991. O Ministério da Saúde, seguro da estratégia de se trabalhar o núcleo familiar como enfoque no desenvolvimento das ações em saúde na atenção básica, iniciou, por volta de 1993 a implementação do Programa Saúde da Família (PSF) no Brasil, hoje denominado Estratégia Saúde da Família (ESF). A implantação deste programa tinha como propósito colaborar decisivamente na consolidação das diretrizes doutrinárias e organizativas do SUS (Sistema Único de Saúde) ${ }^{2}$.

Em 2000 o MS propôs, como estratégia de reorganização da atenção básica odontológica, a inclusão de 
Equipe de Saúde Bucal (ESB) no PSF por meio da Portaria $\mathrm{n}^{\mathrm{o}} 1.444^{3}$, que estabeleceu incentivo financeiro para a reorganização da atenção à saúde bucal aos municípios. No Brasil, a inserção das ações de saúde bucal na Estratégia Saúde da Família (ESF) não ocorreu concomitante a criação desta, o que limitou a integralidade da atenção à saúde, uma vez que desconsiderou que o homem é um ser integral e que sua saúde bucal faz parte de seu contexto bio-psico-social.

Paralelamente a esta inserção, ocorriam discussões acerca da formação em odontologia, que antecederam a conformação do Sistema Único de Saúde - SUS pela constituição federal, e culminou na publicação das Diretrizes Curriculares Nacionais no ano de 2002 (DCN) ${ }^{4}$. Embora tenha ocorrido intensas discussões em torno dos ajustes necessários para a formação do Cirurgião Dentista para o SUS, e que isto tenha sido contemplado nas mudanças publicadas de modo bastante enfático, não foram suficientes para provocar alterações substanciais no perfil desejado e nos objetivos de formação dos cursos de Odontologia ${ }^{5-7}$.

$O$ perfil de formação do profissional cirurgião dentista para contemplar uma abordagem multiprofissional, desejada pela ESF, não se concretizou de modo pleno no período pós publicação das diretrizes curriculares.

O início das atividades da ESF sem a ESB também acarretou prejuízos no processo de integração dos profissionais da equipe de saúde, assim como pode ter determinado formas variadas no processo de implantação das equipes de saúde bucal ${ }^{8}$.

Embora não tenha ocorrido desde o momento inicial, a implementação das ESBs trouxe consigo novas abordagens aos Agentes Comunitários de Saúde (ACS), dentre as quais, a compreensão da saúde bucal como componente da saúde em sua expressão mais ampla9 ${ }^{9}$. Na ESF, o ACS participa ativamente da organização da atenção à saúde familiar em nível da atenção básica, ao realizar o cadastro das famílias e as visitas mensais de acompanhamento das mesmas e de indivíduos e/ou grupos prioritários. Também tem importante papel na divulgação de informações sobre saúde bucal, devendo ser orientado pela referida Equipe ${ }^{10}$.

Frente a isso, o ACS em articulação à ESB e devidamente capacitado, pode contribuir para fortalecer o conhecimento da população no enfrentamento dos problemas de saúde, além de auxiliar a equipe de saúde bucal na identificação das famílias mais vulneráveis que necessitam de ações específicas, bem como melhorar o acesso e a utilização dos serviços primários de saúde, a fim de evitar a assistência odontológica tardia, reduzindo a necessidade de consultas de urgência ${ }^{11}$.

Os autores levantam a hipótese que a inserção tardia da ESB no trabalho multiprofissional da ESF, assim como, a formação acadêmica do cirurgião-dentista calcada no entendimento de saúde bucal como reflexo dos procedimentos clínicos, possa ser um fator limitante no desenvolvimento de atividades de educação, prevenção e vigilância em saúde bucal pelos ACS. O objetivo deste trabalho foi avaliar a percepção dos ACS em relação à saúde bucal da criança, bem como as atividades preventivas e de vigilância em saúde bucal desenvolvidas no âmbito dos domicílios, do município de Francisco Beltrão, cobertos pelas ESBs.

\section{MATERIAL E MÉTODO}

Trata-se de um estudo descritivo, com uma abordagem quantitativa, cujo procedimento metodológico foi o levantamento das informações por meio da aplicação de questionários para os ACS pertencentes à ESF, com ESB. O estudo foi realizado em junho/2016, no município de Francisco Beltrão, localizado na região sudoeste do estado do Paraná, com Índice de Desenvolvimento Humano de 0,77 , população censitária de 78.943 , dos quais 17.527 (22\%) são crianças de 0 a 14 anos de idade, que foi foco deste trabalho no que se refere aos conhecimentos e atividades desenvolvidas pelo ACS. Segundo a Secretaria Municipal de Saúde, o município possui 19 ESF, sendo 16 na zona urbana e 3 na zona rural, das quais apenas 09 equipes possuem ESB, modalidade I, atuando na área urbana num total de 72 ACS

Quanto aos aspectos éticos e metodológicos foi contatado o coordenador de saúde bucal para informá-lo a respeito do objetivo do estudo e de posterior uso dos dados coletados, a fím de obter autorização para a realização do mesmo. O trabalho foi conduzido dentro dos padrões exigidos pela Resolução 196/CNS e aprovado pelo Comitê de Ética em Pesquisa em Seres Humanos da Universidade Paranaense - UNIPAR, sob o protocolo número 56379416.9.0000.0109.

Foram incluídos no estudo os ACS que aceitaram participar e que estavam presentes no dia da aplicação do questionário, oportunidade em que foram informados quanto à finalidade e ao sigilo das informações coletadas, assinando o Termo de Consentimento Livre e Esclarecido, em caso de concordância. Para a coleta dos dados, foi utilizado um questionário estruturado, autoaplicável, composto por questões referentes à percepção dos ACS com relação à saúde bucal das crianças de 0 a 14 anos, desenvolvimento de ações preventivas e de vigilância à saúde durante suas visitas domiciliares. A formulação das perguntas do questionário baseou-se e em estudo semelhante realizado com ACS do município de São José do Rio Preto - $\mathrm{SP}^{12}$. O preenchimento do questionário foi realizado pelo sujeito pesquisado, sempre numa sala da Unidade Básica de Saúde sob supervisão do pesquisador, de maneira que os mesmos fossem respondidos de forma individualizada pelos ACS. As questões abordavam conhecimentos de saúde bucal relacionados a crianças de 0 a 14 anos e apresentavam-se de forma dicotômicas de sim e não e de respostas de múltiplas escolhas. As questões relacionadas às atividades preventivas e de vigilância em saúde bucal desenvolvidas no âmbito familiar foram formuladas com respostas objetivas em série temporal, com a amplitude de tempo (anualmente, mensalmente, semanalmente, diariamente e nunca).

Os ACS tiveram assegurada sua privacidade quanto aos dados coletados, o caráter confidencial de suas respostas e seu direito de não-identificação. Após a categorização, os dados coletados foram digitados em planilha do programa Excel, transportados e analisados no programa estatístico Bioestat, versão 5.3. Os testes estatísticos utilizados para verificação de associação entre as variáveis foram o Wilcoxon-Mann-Whitney e Teste Exato de Fisher com nível de significância de $5 \%$.

\section{RESULTADOS}

O município de Francisco Beltrão possui 16 ESF na área urbana, sendo que destas, 09 estão trabalhando com ESB. A totalidade de agentes pertencentes a estas nove equipes somam 72 , sendo que 16 agentes $(22,2 \%)$ não responderam à aplicação do formulário por motivos diversos (doença, atestado, férias e falta no serviço no dia da aplicação do formulário). 
Os resultados quanto à capacitação dos agentes comunitários sobre saúde bucal são apresentados na Tabela 1 e para $71,4 \%$ deles, este assunto nunca foi abordado o que pode ser percebido quando verificamos que questões relativas a doença cárie, gengivite e fluorose não eram de conhecimento da maioria dos agentes pesquisados. A identificação do início da doença cárie foi respondida corretamente por apenas 30,3\% dos ACS, sendo a fluorose dentária, a questão menos conhecida pelos agentes.

Entretanto, quando questionados sobre a quantidade de dentifrício a ser utilizada durante uma escovação para uma criança com menos de dois anos de idade, a maioria $(66 \%)$ respondeu corretamente. Grande parte dos ACS $(58,9 \%)$ desconhece o momento ideal para começar a escovação com dentifrício, contudo, sabem que esta deve ser supervisionada pelos pais até a idade entre 7 a 8 anos. A quantidade correta de creme dental utilizada durante a escovação, bem como, a supervisão por um adulto são fatores importantíssimos na prevenção da fluorose dentária (Tabela 1).

Tabela 1. Realização de oficinas de capacitação e conhecimento dos Agentes Comunitários de Saúde sobre doença cárie, gengivite e fluorose em crianças. Francisco Beltrão, PR, 2016

\begin{tabular}{|c|c|c|}
\hline Questões & $n$ & $\%$ \\
\hline \multicolumn{3}{|l|}{ Em seu trabalho como Agente } \\
\hline \multicolumn{3}{|l|}{$\begin{array}{l}\text { Comunitário de Saúde você tem recebido } \\
\text { capacitação sobre saúde bucal? }\end{array}$} \\
\hline Sim & 16 & 28,56 \\
\hline Não & 40 & 71,42 \\
\hline \multicolumn{3}{|l|}{$\begin{array}{l}\text { Você saberia identificar cárie por meio } \\
\text { de exame bucal em uma criança? }\end{array}$} \\
\hline Sim & 34 & 60,71 \\
\hline Não & 22 & 39,28 \\
\hline \multicolumn{3}{|l|}{ O início de uma cárie dentária é: } \\
\hline $\begin{array}{l}\text { Quando há uma pequena cavidade na superfície } \\
\text { do dente }\end{array}$ & 26 & 46,42 \\
\hline $\begin{array}{l}\text { Quando há uma mancha branca na superfície do } \\
\text { dente }\end{array}$ & 17 & 30,35 \\
\hline Não saberia dizer & 13 & 23,21 \\
\hline \multicolumn{3}{|l|}{ Você sabe o que é uma gengivite? } \\
\hline Sim & 27 & 48,21 \\
\hline Não & 29 & 51,78 \\
\hline \multicolumn{3}{|l|}{ Você sabe o que é fluorose dentária? } \\
\hline Sim & 15 & 26,78 \\
\hline Não & 41 & 73,21 \\
\hline \multicolumn{3}{|l|}{$\begin{array}{l}\text { Qual quantidade de creme dental você } \\
\text { colocaria para escovar os dentes de uma } \\
\text { criança de dois anos? }\end{array}$} \\
\hline Tamanho de um grão de arroz & 37 & 66,07 \\
\hline Tamanho de um grão de ervilha & 17 & 30,35 \\
\hline Toda extensão das cerdas da escova & 02 & 3,57 \\
\hline \multicolumn{3}{|l|}{$\begin{array}{l}\text { Qual o momento ideal para começar a } \\
\text { escovação com creme dental? }\end{array}$} \\
\hline Antes do aparecimento do primeiro dente & 11 & 19,64 \\
\hline A partir do aparecimento do primeiro dente & 23 & 41,07 \\
\hline $\begin{array}{l}\text { Quando a criança já souber cuspir } \\
\text { completamente a espuma do creme dental }\end{array}$ & 19 & 33,92 \\
\hline Outros & 03 & 5,35 \\
\hline \multicolumn{3}{|l|}{$\begin{array}{l}\text { Até que idade você acredita que os pais } \\
\text { devam supervisionar a escovação do seu } \\
\text { filho? }\end{array}$} \\
\hline ( ) 2 anos & 01 & 1,78 \\
\hline ( ) 3 a 4 anos & oo & 0,00 \\
\hline ( ) 5 a 6 anos & 04 & 7,14 \\
\hline ( ) 7 a 8 anos/mais & 51 & 91,07 \\
\hline
\end{tabular}

Pode-se observar em nosso estudo, que nas nove UBS em que foram coletadas as informações, existiam ACS capacitados e ACS que não haviam sido capacitados quanto aos cuidados necessários para a promoção e manutenção da saúde bucal.

Para verificar se a presença de ACS capacitados era estatisticamente significante optou-se pela utilização do teste de aleatorização considerando a estatística do teste de Wilcoxon-Mann-Whitney (teste não-paramétrico).

As hipóteses testadas foram: $\mathrm{H} 0=$ as duas amostras são provenientes de populações idênticas ou da mesma população versus $\mathrm{H} 1=$ as duas amostras são provenientes de populações diferentes.

Foram realizadas 10.000 aleatorizações, através do $\mathrm{R}$ e o p-valor (do teste de aleatorização) foi 0,0086 . Como este valor é menor que o nível de significância $(\alpha=0,05)$ então, rejeitou-se $\mathrm{H} 0$ ao nível de $5 \%$ de significância, ou seja, as amostras são provenientes de populações diferentes, o que vale afirmar que a presença de ACS capacitados foi estatisticamente significante.

Observou-se que não houve associação estatisticamente significante entre as variáveis: capacitação e conhecimento sobre a doença cárie na sua fase inicial $(p=0,1940)$, capacitação e conhecimento sobre gengivite ( $p$ $=0,3837)$, capacitação e conhecimento sobre fluorose $(\mathrm{p}=$ $0,4421)$, capacitação e conhecimento sobre a quantidade correta de dentifrício $(\mathrm{p}=0,5236)$ e capacitação e conhecimento sobre o momento ideal para se iniciar a escovação com uso de dentifrício $(p=0,2869)$ (Tabela 2$)$.

Tabela 2. Associação entre as variáveis capacitação, conhecimento sobre: cárie dentária, fluorose, quantidade de dentifrício e momento ideal para o início da escovação. Francisco Beltrão, PR, 2016

\begin{tabular}{lccc}
\hline \multicolumn{1}{c}{ Variáveis } & \multicolumn{2}{c}{ Capacitação } & p \\
\hline $\begin{array}{l}\text { Conhecimento sobre } \\
\text { cárie - fase inicial }\end{array}$ & & & \\
Sim & 07 & 11 & \\
Não & 09 & 29 & $\mathrm{p}=0,1940$ \\
& & & \\
$\begin{array}{l}\text { Conhecimento sobre } \\
\text { gengivite }\end{array}$ & 12 & 04 & \\
Sim & 33 & 07 & $\mathrm{p}=0,3837$ \\
Não & & & \\
Conhecimento sobre & & & \\
$\begin{array}{l}\text { a quantidade correta } \\
\text { de dentifrício }\end{array}$ & & & \\
Sim & 11 & 26 & $\mathrm{p}=0,5236$ \\
Não & 05 & 14 & \\
$\begin{array}{l}\text { Conhecimento sobre } \\
\text { o início da escovação } \\
\text { com dentifrício }\end{array}$ & & & \\
Sim & & & \\
Não & 08 & 15 & $\mathrm{p}=0,2869$ \\
\hline Teste Exato de Fisher (p< 0,05$)$ & & &
\end{tabular}

Pode-se verificar que a frequência com que a equipe de saúde bucal acompanha os agentes durante as visitas familiares é bastante baixa, chegando a 58,9\% nunca terem realizado esta atividade. Os resultados da Tabela 3 indicam que as atividades preventivas que poderiam ser realizadas no âmbito da família, pelos ACS, e que resultariam em aumento de cobertura das ações odontológicas, não são desenvolvidas.

Foi possível constatar que atividades preventivas, tais como a supervisão da escovação, evidenciação do biofilme e bochechos fluoretados nunca foram realizadas, respectivamente por $96,4 \%, 100 \%$ e $100 \%$ dos agentes comunitários que fizeram parte desta pesquisa. Ações de vigilância, como verificar acesso e necessidade de dentifrício por parte das famílias, foi realizado por $21,5 \%$ dos agentes comunitários, entretanto, esta importante ação de vigilância na prevenção da doença cárie nunca foi realizada por $78,5 \%$ deles. $\mathrm{O}$ encaminhamento para o 
tratamento odontológico já foi realizado por dois terços dos agentes comunitários pesquisados (Tabela 3 ).

Tabela 3. Atividades preventivas e de vigilância em saúde bucal desenvolvidas no âmbito familiar. Francisco Beltrão, PR, 2016

\begin{tabular}{|c|c|c|}
\hline Questões & $\mathbf{n}$ & $\%$ \\
\hline \multicolumn{3}{|c|}{$\begin{array}{l}\text { Com que frequência a Equipe de Saúde Bucal } \\
\text { acompanha o trabalho do Agente Comunitário } \\
\text { de Saúde? }\end{array}$} \\
\hline Anualmente & 06 & 10,71 \\
\hline Mensalmente & 03 & 5,35 \\
\hline Semanalmente & 09 & 16,07 \\
\hline Diariamente & 05 & 8,92 \\
\hline Nunca & 33 & 58,92 \\
\hline \multicolumn{3}{|c|}{$\begin{array}{l}\text { Você supervisiona a escovação dentária das } \\
\text { crianças durante as visitas domiciliares? }\end{array}$} \\
\hline Anualmente & 02 & 3,57 \\
\hline Mensalmente & 0 & 0 \\
\hline Semanalmente & o & $\mathrm{o}$ \\
\hline Diariamente & o & 0 \\
\hline Nunca & 54 & 96,42 \\
\hline \multicolumn{3}{|c|}{$\begin{array}{l}\text { Você utiliza evidenciador de placa bacteriana } \\
\text { durante as visitas domiciliares? }\end{array}$} \\
\hline Anualmente & o & o \\
\hline Mensalmente & o & 0 \\
\hline Semanalmente & o & o \\
\hline Diariamente & o & $\mathrm{o}$ \\
\hline Nunca & 56 & 100 \\
\hline \multicolumn{3}{|c|}{$\begin{array}{l}\text { Você realiza bochechos fluoretados nas } \\
\text { crianças durante as visitas domiciliares? }\end{array}$} \\
\hline Anualmente & o & o \\
\hline Mensalmente & o & 0 \\
\hline Semanalmente & o & 0 \\
\hline Diariamente & o & o \\
\hline Nunca & 56 & 100 \\
\hline \multicolumn{3}{|c|}{$\begin{array}{l}\text { Com que frequência você verifica durante as } \\
\text { visitas domiciliares o acesso (presença) e } \\
\text { necessidade (falta) de creme dentário pelas } \\
\text { famílias? }\end{array}$} \\
\hline Anualmente & 0 & o \\
\hline Mensalmente & 03 & 5,35 \\
\hline Semanalmente & 01 & 1,78 \\
\hline Diariamente & 08 & 14,28 \\
\hline Nunca & 44 & 78,57 \\
\hline \multicolumn{3}{|c|}{$\begin{array}{l}\text { Com que frequência você realiza } \\
\text { encaminhamentos para o tratamento } \\
\text { odontológico na Unidade Básica de Saúde } \\
\text { durante suas visitas domiciliares? }\end{array}$} \\
\hline Anualmente & 02 & 3,57 \\
\hline Mensalmente & 11 & 19,64 \\
\hline Semanalmente & 6 & 10,71 \\
\hline Diariamente & 18 & 32,14 \\
\hline Nunca & 19 & 33,92 \\
\hline
\end{tabular}

\section{DISCUSSÃO}

O presente trabalho teve o intuito de verificar os conhecimentos e o desenvolvimento das práticas preventivas e de vigilância em saúde bucal realizadas pelos agentes comunitários de saúde durante as visitas domiciliares, tendo como foco a saúde bucal da criança de 0 a 14 anos.

O fato das equipes de saúde bucal passar a fazer parte da Estratégia Saúde da Família, em âmbito nacional a partir do início do ano de 2001, não deve ser considerado um contratempo para que os ACS exerçam seu trabalho de forma multiprofissional com a equipe de saúde bucal, quando treinados. Esse fato é corroborado pelo estudo de Frazão e Marques, os quais verificaram mudanças significativas nos conhecimentos de saúde bucal por parte de mulheres e de mães da área de atuação dos agentes, que foram capacitados a realizar Educação em Saúde junto à comunidade ${ }^{11}$.

Pesquisas realizadas quanto à opinião dos ACS acerca da competência de realização de atividades em saúde bucal, têm demonstrado que quase a totalidade acredita que orientações sobre saúde bucal na comunidade poderia ser de sua competência, revelando a possibilidade de atuação destes profissionais no assunto ${ }^{13-16}$. Na presente pesquisa, pode-se constatar que a maioria, $71,4 \%$, dos agentes não foram capacitados para poder orientar sua comunidade sobre a saúde bucal das crianças, durante suas visitas domiciliares. Resultado semelhante foi encontrado no estudo realizado em quatro municípios de pequeno porte $(8.000$ a 26.000 habitantes), onde cerca de $80 \%$ dos ACS pesquisados não foram capacitados por equipes de saúde bucal ${ }^{13}$. Pires ${ }^{14}$, também ao pesquisar o conhecimento dos ACS sobre saúde bucal em um município do interior de São Paulo, apurou que 65,38\% dos ACS não tiveram capacitação sobre o tema.

Por outro lado, há trabalhos que demonstram que ACS foram capacitados e assistiram palestras sobre saúde bucal na unidade de saúde, mas quando avaliados sobre seus conhecimento e atitudes sobre a promoção da saúde bucal, os mesmos ainda relatam que possuem muita dificuldade em realizar suas atribuições por não terem uma programação definida para a comunidade ${ }^{15,17}$. No presente estudo, também foi possível verificar que não há uma diferença estatística entre os ACS que disseram ter recebido capacitação e conhecimento sobre as questões abordadas, quando comparado aos ACS que não receberam capacitação, demonstrando a necessidade de uma educação contínua e maior integração das ESB com as equipes multiprofissionais das unidades de saúde estudadas.

A falta de associação estatística entre a capacitação e conhecimento na área de saúde bucal, sugere a necessidade de educação continuada como forma de aperfeiçoamento das habilidades e conhecimento da realidade das condições de saúde bucal em que estão inseridos, visando uma prática mais atuante, que venha contribuir com a proposta da estratégia saúde da família.

Quando não há capacitação dos ACS, é de se esperar que os conhecimentos adquiridos ao longo de sua vida possam distinguir do conhecimento técnico científico. Isto pode ser comprovado quando a maioria $(60,7 \%)$ disse saber identificar a doença cárie por meio de um exame bucal, entretanto, somente $30,3 \%$ responderam corretamente que o início da cárie é quando há uma mancha branca sobre a superfície do dente. A maioria dos agentes, neste estudo, acredita que a cavidade na superfície do dente é o início da cárie dentária. Pensando em uma atuação mais abrangente pelo ACS, elevar o domínio sobre informações e conhecimentos sobre a doença cárie pode contribuir para aumentar suas habilidades no controle de determinantes de risco e, maior socialização de informações para as famílias. Esta atuação, além de intensificar as ações preventivas a estes grupos, podem ajudar a equipe de saúde bucal na identificação das famílias mais vulneráveis que necessitam melhorar o acesso e a utilização dos serviços básicos de saúde, e assim evitar a assistência odontológica tardia e consulta de urgência.

O fator que ocasiona a gengivite é desconhecido por praticamente metade $(51,7 \%)$ dos ACS entrevistados e no que se refere à fluorose dentária, a falta de conhecimento sobre este agravo chegou a $73,2 \%$. Pesquisas de âmbito nacional $^{18-19}$ têm demonstrado um aumento de fluorose nas crianças brasileiras, sendo que conhecimentos sobre os fatores de risco e proteção pelos ACS poderiam fazer parte das atividades de orientação às famílias com crianças de baixa idade. Uma constatação positiva pode ser observada com relação à quantidade do dentifrício a ser utilizada para realizar uma escovação em criança com dois anos de idade, onde $66,0 \%$ e $30,3 \%$ disseram que a quantidade de um grão de arroz e de ervilha, respectivamente, seria adequado.

Os autores deste estudo não compartilham da informação sobre o Guia Prático do Agente Comunitário de Saúde, formulado pelo Ministério da Saúde, sobre o uso do 
dentifrício em crianças de baixa idade. O documento orienta que o creme dental com flúor só deverá ser utilizado quando a criança souber cuspir completamente o seu excesso, pois elas podem engolir essa pasta e vir a ter um problema conhecido como fluorose, que é o aparecimento de manchas esbranquiçadas e/ou má formação dentária ${ }^{20}$. Os autores defendem que o creme dental fluoretado deve ser usado desde a erupção dos primeiros dentes, na quantidade correta, pois a desorganização do biofilme pela escovação, associada à disponibilidade de fluoreto na cavidade bucal é considerada estratégia importante na prevenção da cárie.

Os ACS podem, no que refere a saúde bucal de crianças, colaborar de forma significativa na orientação e ações de prevenção e vigilância relacionadas à cárie, gengivite e fluorose dentária. No que refere à cárie e gengivite, o seu reconhecimento por meio do exame visual pelo agente comunitário devidamente capacitado, poderia facilitar o acolhimento e acesso ao tratamento curativo bem como, direcionar o desenvolvimento de ações preventivas e de vigilância no âmbito do domicílio. As atividades preventivas sugeridas pelos autores podem ser a identificação do biofilme com uso de evidenciador, supervisão da escovação, fluorterapia para as crianças com risco ou atividade de cárie, verificação das condições de uso da escova dental e armazenamento, bem como, dependendo da estratégia adotada na atenção básica, provimento de escova e dentifrício para famílias com maior vulnerabilidade social.

Os agentes capacitados para orientar as famílias sobre questões de saúde bucal poderiam aumentar de forma significativa a cobertura de ações preventivas e de vigilância à saúde bucal, quando comparado à equipe de saúde da família trabalhando de forma isolada. Uma ESB, geralmente é composta por dois profissionais, no máximo três, enquanto que os agentes comunitários de uma equipe saúde, locado numa unidade, podem chegar a doze, sendo que estes realizam visitas domiciliares com frequência muito maior que a equipe de saúde bucal. $\mathrm{O}$ trabalho multiprofissional no plano da oferta e organização dos serviços de saúde público para o cirurgião-dentista, é um desafio até mesmo pela sua própria formação acadêmica cuja visão predominante de saúde ainda é pautada sob o ponto de vista biológico, centrada no tratamento individual, baseado nos instrumentos e equipamentos da clínica odontológica e principalmente voltada para o exercício privado da profissão.

Apesar dos diversos incentivos por parte do Ministério da Saúde e do Ministério de Educação, de discussões intensas e permanentes pelas diversas entidades da classe odontológica, tais como, Associação Brasileira de Odontologia, Associação Brasileira de Ensino Odontológico, Conselho Federal de Odontologia, as mudanças necessárias para a implementação das DCN ainda não configuram a realidade dos cursos no país ${ }^{5-7}$.

Para os autores deste trabalho estas dificuldades de implementação das DCN interferem diretamente na forma de interação da ESB com os demais membros da ESF. Tais dificuldades podem impossibilitar o princípio de trabalho em equipe preconizado pela estratégia, bem como o de integralidade das ações e, por vezes, inviabilizando a adoção de práticas inovadoras de educação continuada. Diante deste quadro, alternativas de indução de mudanças via sistema de avaliação dos cursos podem resultar em melhor implementação das DCN e assim, melhorar as estratégias de atuação da ESB quanto a educação continuada dos $\mathrm{ACS}^{7}$.

À medida que existem inúmeras ESB na ESF já implantadas no Brasil, a educação continuada deve considerar inicialmente, as próprias equipes de ESB, de modo que estas possam conduzir a contento a educação continuada dos ACS. Isso impõe aos educadores e profissionais de saúde que seja dada uma maior relevância às práticas ambulatoriais e comunitárias, a diversas formas de integração de ensino com serviços e de formação de redes de instituições de ensino ${ }^{21}$. Outra estratégia para capacitação deve ser associada a um acompanhamento periódico por parte do Cirurgião-dentista da unidade, a fim de esclarecer possíveis dúvidas, e ainda possibilitar o reforço dos conhecimentos em saúde bucal adquiridos pelos agentes no momento da capacitação ${ }^{22}$.

Inúmeros cursos de Odontologia brasileiros passaram por reformulação de seus projetos pedagógicos (PPP), tendo como meta a formação de um cirurgião-dentista generalista, centrado nas necessidades de saúde da população e na política de saúde vigente no País. Dentre as ações desenvolvidas nestes processos inovadores de PPP, destacase a inserção dos acadêmicos na Estratégia Saúde da Família (ESF) em comunidades de alta vulnerabilidade social. Dentre as práticas inovadoras, destacam-se as atividades direcionadas à comunidade tais como, visitas domiciliares, atividades coletivas de promoção de saúde, programas preventivos em equipamentos sociais, participação em reuniões da equipe da ESF, participação efetiva no Conselho Local de Saúde e atendimento odontológico. A inserção dos acadêmicos na realidade de vida das pessoas tem permitido reflexões diversas sobre os determinantes do processo saúdedoença $^{23}$

É imprescindível a formação de futuros profissionais da saúde sensibilizados com as necessidades da população e pautados nas diretrizes da política nacional de saúde bucal, bem como das Diretrizes Curriculares da Odontologia, para que possam desenvolver um trabalho inserido na equipe multiprofissional de forma que, entre as atribuições do cirurgião-dentista, esteja a capacitação dos agentes comunitários para o desenvolvimento de ações de saúde bucal durante as visitas domiciliares, buscando dessa forma mais integralidade na prestação dos serviços odontológicos e equidade na sua oferta.

\section{CONCLUSÃO}

Com o desenvolvimento desta pesquisa podemos concluir que a odontologia de Francisco Beltrão não tem capacitado de forma continua os ACS de forma a colaborar com o trabalho multiprofissional buscando uma maior integralidade da atenção à saúde.

Não houve uma preocupação por parte da ESB em direcionar ao núcleo familiar ações prevenção e de vigilância à saúde bucal por meio do ACS durante suas visitas domiciliares.

\section{REFERÊNCIAS}

1. Andrade LOM, Barreto ICHC, Goya N, Martins-Jr T. Organização da atenção básica e Estratégia Saúde da Família no município de Sobral - Ceará - Brasil: principais avanços e desafios na perspectiva de produzir mudanças positivas na saúde. In: Seclen J, Fernandes AS, organizadores. Experiências e desafios da atenção básica e saúde familiar: caso Brasil. Brasília: OPAS; 2004. p.143-79. (Serie técnica Projeto de Desenvolvimento de Sistemas e Serviços de Saúde, 8). Acesso em: 06 de outubro de 2016. Disponível em: http://new.paho.org/hq/dmdocuments/2010/ap_Atencion _Primaria_Salud_Familiar-Brasil.pdf 
2. Viana ALA, Poz MRD. A reforma do sistema de saúde no Brasil e o programa de Saúde da Família. PHYSIS: Rev Saúde Coletiva. 2005; 15(Supl.):S225-64.

3. Brasil. Ministério da Saúde. Portaria $n^{\circ} 1.444,28$ de dezembro de 2000. Estabelece incentivo financeiro para a reorganização da atenção à saúde bucal prestada nos municípios por meio do Programa de Saúde da Família. Diário Oficial da República Federativa do Brasil. Brasília, DF. 29 de dezembro 2000. Acesso em: 06 de outubro de 2016. Seção 1, (85) disponível em: www.mp.go.gov.br/portalweb/hp/2/docs/portaria1444_2 8_12_00.pdf

4. Brasil. Conselho Nacional de Educação. Câmara de Educação Superior. Diretrizes curriculares nacionais do curso de Graduação em Odontologia. Diário Oficial da União, Brasília, 2002;1:10.

5. Freitas SFT, Calvo MCM, Lacerda JT. Saúde coletiva e novas diretrizes curriculares em odontologia: uma proposta para graduação. Trab Educ Saúde. 2012; 10(2):223-34.

6. Morita MC, Kriger L. Mudanças nos cursos de Odontologia e a interação com o SUS. Rev Abeno. 2004; 4(1):17-21.

7. Brasil. Ministério da Saúde. Ministério da Educação. A aderência dos cursos de graduação em enfermagem, medicina e odontologia às diretrizes curriculares nacionais / Ministério da Saúde, Ministério da Educação. - Brasília: Ministério da Saúde, 2006. 162 p.: il. - (Série F. Comunicação e Educação em Saúde).

8. Baldani MH, Fadel CB, Possamai T, Queiroz MGS. A inclusão da odontologia no Programa Saúde da Família no Estado do Paraná, Brasil. Cad Saúde Pública. 2005; 21(4):1026-35.

9. Souza DS, Cury JA, Caminha JAN, Ferreira MA, Tomita NE, Narvai PC. A Inserção da saúde bucal no Programa de Saúde da Família. Rev Bras Odontol. 2001; 2(2):7-29.

10. Brasil. Ministério da Saúde. Departamento de Atenção Básica. Saúde bucal. Brasília: Ministério da Saúde; 2006. 92p. (Cadernos de Atenção Básica; 17) (Série A. Normas e Manuais Técnicos). Acesso em 13 de outubro de 2016. Disponível em: http://189.28.128.100/dab/ docs/publicacoes/cadernos_ab/abcad17.pdf

11. Frazão P, Marques DSC. Influência de agentes comunitários de saúde na percepção de mulheres e mães sobre conhecimentos de saúde bucal. Cien Saúde Colet. 2006; 11(1):131-44.

12. Terreri ALM, Ribeiro MTF, Barbosa PRA, Lisboa SGS, Marconi PMC. A percepção dos agentes comunitários sobre a saúde bucal das crianças e o desenvolvimento das ações preventivas. Rev Paul Odontol. 2011; 33:4551 .

13. Moura MS, Carvalho CJ, Amorim JTC, Marques MFSS, Moura LFAD, Mendes RF. Perfil e práticas de saúde bucal do agente comunitário de saúde em municípios piauienses de pequeno porte. Cien Saúde Colet. 2010; 15(1):1487-95.

14. Pires ROM, Neto FL, Lopes JB, Bueno SMV. O conhecimento dos agentes comunitários sobre saúde bucal: uma perspectiva sobre deficiências em educação no PSF. Cienc Cuid Saúde. 2007; 6(3):325-34.

15. Mockdeci HR, Souza TS, Rodrigues CM, Leite ICG. Capacitação em saúde bucal de Agentes Comunitários em Juiz de Fora- MG: a efetividade do programa. HU Revista. 2013; 39(3/4):45-52.
16. Koyashiki GAK, Alves-Souza RA, Garanhani ML. O trabalho em saúde bucal do Agente Comunitário de Saúde em Unidades de Saúde da Família. Cien. Saúde Colet. 2008; 13(4):1343-54.

17. Levy FM, Matos PES, Tomita NE. Programa de agentes comunitários de saúde: a percepção de usuários e trabalhadores da saúde. Cad. Saúde Pública. 2004; 20(1):197-203

18. Brasil. Ministério da Saúde. Projeto SB Brasil 2010: Pesquisa Nacional de Saúde Bucal - resultados principais. Brasília: Ministério da Saúde; 2011. Disponível em: http://dab.saude.gov.br/CNSB/ sbbrasil/arquivos/projeto_sb2010_relatorio_final.pdf

19. Brasil. Ministério da Saúde. Departamento de Atenção Básica. Projeto SB Brasil 2003: condições de saúde bucal da população brasileira 2002-2003: resultados principais. Brasília: Ministério da Saúde, abril, 2004. Disponível em: http://bvsms.saude.gov.br/bvs/ publicacoes/condicoes_saude_bucal.pdf

20. Brasil. Ministério da Saúde. Secretaria de Atenção à Saúde. Departamento de Atenção Básica. Guia prático do agente comunitário de saúde / Ministério da Saúde, Secretaria de Atenção à Saúde, Departamento de Atenção Básica. - Brasília: Ministério da Saúde, 2009. Disponível em: http://dab.saude.gov.br/docs/ publicacoes/geral/guia_acs.pdf

21. Cardoso ACC, Santos Jr RQ, Souza LEP, Barbosa MBCB. Inserção da equipe de saúde bucal no PSF: um desafio para a melhoria da qualidade de atenção à saúde. Rev Baiana Saúde Públ. 2002; 26(1-2):94-8.

22. Moura MS, Carvalho CJ, Amorim JTC, Marques MFSS, Moura LFAD, Mendes RF. Perfil e práticas de saúde bucal do agente comunitário de saúde em municípios piauienses de pequeno porte. Ciên Saúde Colet. 2010; 15(1):1487-95.

23. Hayacibara MF, Lolli LF, Terada RSS, Hidalgo MM, Bispo CGC, Terada HH, et al.. Experiência de clínica ampliada em odontologia na Universidade Estadual de Maringá. Rev Bras Educ Med. 2012; 36(1,Suppl 2):17883.

\section{CONFLITO DE INTERESSES}

Os autores declaram não haver conflitos de interesse.

\section{AUTOR PARA CORRESPONDÊNCIA}

\section{Daniela Pereira Lima}

dani.pl@hotmail.com

Submetido em 02/03/2017 Aceito em 19/03/2017 